\title{
ORDINARY DICHOTOMY AND PERTURBATIONS OF THE COEFFICIENT MATRIX OF THE LINEAR IMPULSIVE DIFFERENTIAL EQUATION
}

\author{
N.V. MILEV and D.D. BAINOV \\ PLOVDIV UNIVERSITY \\ "PAISSII HILENDARSKI" \\ P.O. Box 45 \\ 1504 Sofia \\ Bulgaria \\ (Received January 17, 1990 and in revised form April 22, 1991)
}

\begin{abstract}
In the present paper it is proved that the ordinary dichotomy is preserved under perttriations of the coefficient matrix of the linear impulsive differential equation.
\end{abstract}

KEY WORDS AND PHRASES. Impulsive differential equation, integral equation and ordinary dichotomy.

1991 AMS SUBJECT CLASSIFICATION CODE. 34G11

\section{INTRODUCTION.}

In relation to numerous applications in science and technology, in the last five years the theory of impulsive differential equations has developed intensively [3]-[6] and [10]. In the present paper one of the important properties of the ordinary dichotomy for linear impulsive differential equations is studied, namely that it is not destroyed under small perturbations of the coefficient matrix. We shall note that analogous questions about ordinary differential equations were considered in [7], [1], [2], [9].

\section{PRELIMINARY NOTES.}

Let $t_{0}<t_{1}<\ldots<t_{i}<\ldots, \lim t_{i}=\infty$ as $i \rightarrow \infty$, be a given sequence of real numbers. Consider the linear differential equation with impulses at fixed moments

$$
\frac{d x}{d t}=A(t) x, \quad t \neq t_{i} ; x\left(t_{i}+0\right)=B_{i} x\left(t_{i}\right), i=1,2 \ldots
$$

where the $(n \times n)$-coefficient matrix $A(t)$ is piecewise continuous in the interval $\left[t_{o},+\infty\right)$ with points of discontinuity of the first kind at $t=t_{i}$ and the impulse matrices $B_{i}, i=1,2, \ldots$, are constant. The underlying vector space $E$ is $\mathbb{R}^{n}$ or $\mathbb{C}^{n}$.

REMARK 1. For $t \in\left[t_{i}+0, t_{i+1}\right]$ the fundamental matrix $X(t)$ of equation (2.1) admits the representation

$$
X(t)=U(t) U^{-1}\left(t_{i}+0\right) B_{i} U\left(t_{i}\right) U^{-1}\left(t_{i-1}+0\right) B_{i-1} \ldots B_{1} U\left(t_{1}\right) U^{-1}\left(t_{o}\right)
$$

where $U(t)$ is the fundamental matrix of the equation $\frac{d x}{d t}=A(t) x$. The matrix $X(t)$ is continuously differentiable for $t \neq t_{i}$ with points of discontinuity of the first kind at $t=t_{i}$, i.e. $X\left(t_{i}+0\right)=B_{i} X\left(t_{i}\right)$. The fundamental matrix $X(t)$ is invertible if and only if the impulse matrices $B_{i}, i=1,2, \ldots$, are nonsingular.

Together with equation (2.1) we shall consider the perturbed equation

$$
\frac{d x}{d t}=(A(t)+\widetilde{A}(t)) x, \quad t \neq t_{i} ; x\left(t_{i}+0\right)=B_{i} x\left(t_{i}\right), i=1,2, \ldots
$$


where the matrix $\widetilde{A}(t)$ is piecewise continuous on the interval $\left[t_{o},+\infty\right)$ with points of discontinuity of the first kind for $t=t_{i}, i=1,2, \ldots$.

Let $\tau_{o}$ be a fixed real number, $\tau_{o} \geq t_{o}$.

DEFINITION 1 ([8]). The subspace $Y$ of the underlying vector space $E$ induces an ordinary dichotomy of the solutions of equation (2.1) on the interval $\left[\tau_{o},+\infty\right)$ if for some subspace $Z$ supplementary to $Y$ there exists a constant $N$ such that all solutions $x, y, z$ of equation (2.1) for which $x=y+z, y\left(\tau_{o}\right) \in Y$ and $z\left(\tau_{o}\right) \in Z$ satisfy the conditions

$$
|y(t)| \leq N|x(s)| \text { for } t \geq s \geq \tau_{o} \text { and }|z(t)| \leq N|x(s)| \text { for } s \geq t \geq \tau_{o}
$$

When the fundamental matrix $X(t)$ is invertible, Definition 1 can be written down in the following form.

DEFINITION 2 ([8]). The subspace $Y$ of the underlying vector space $E$ induces an ordinary dichotomy of the solutions of equation (2.1) on the interval $\left(\tau_{o},+\infty\right)$ if for some projector $P\left(P^{2}=P\right)$ with range $R(P)=Y$ there exists a constant $N$ such that

$$
\begin{gathered}
\left|X(t) X^{-1}\left(\tau_{o}\right) P X\left(\tau_{o}\right) X^{-1}(s)\right| \leq N \quad \text { for } t \geq s \geq \tau_{o} \\
\left|X(t) X^{-1}\left(\tau_{o}\right)(I-P) X\left(\tau_{o}\right) X^{-1}(s)\right| \leq N
\end{gathered}
$$

where $I$ stands for the unit matrix.

DEFINITION 3. Let $P$ be a projector $\left(P^{2}=P\right)$. The function

$$
G(t, s)=\left\{\begin{array}{lr}
X(t) P X^{-1}(s) & \text { for } t \geq s \geq t_{o} \\
X(t)(P-I) X^{-1}(s) & \text { for } s>t \geq t_{o}
\end{array}\right.
$$

will be called Green's function for equation (2.1).

We shall use the following properties of Green's function which are verified immediately:

and

$$
\frac{\partial G(t, s)}{\partial t}=A(t) G(t, s) \text { for } t \neq s, G\left(t_{i}+0, t\right)=B_{i} G\left(t_{i}, t\right)
$$

$$
G(t, t+0)-G(t, t-0)=-I \quad \text { for } t \neq t_{i}, \quad i=1,2, \ldots
$$

\section{MAIN RESULTS.}

THEOREM 1. Let the impulse matrices $B_{i}, i=1,2, \ldots$, of equation $(2.1)$ be nonsingular and let the subspace $Y$ induce an ordinary dichotomy of the solutions of equation (2.1) on the interval $\left[t_{o},+\infty\right)$ with a projector $P$ and a constant $N$. If

$$
\int_{t_{o}}^{\infty}|\widetilde{A}(\theta)| d \theta=K<\frac{1}{N(2 N+1)}
$$

then the perturbed equation (2.2) also has an ordinary dichotomy on the interval $\left[t_{o},+\infty\right)$.

PROOF. Let $X(t)$ be the fundamental matrix of equation (2.1) for which $X\left(t_{o}\right)=I$. The bounded solutions $y(t)$ of equation (2.2) are just the bounded solutions of the integral equation

$$
y(t)=X(t) \eta+\int_{t_{o}}^{\infty} G(t, \theta) \widetilde{A}(\theta) y(\theta) d \theta, \quad \eta \in Y,
$$


since for $t \neq t_{i}$

$$
\begin{aligned}
\frac{d y(t)}{d t} & =X^{\prime}(t) \eta+\frac{d}{d t}\left(\int_{t_{o}}^{t} G(t, \theta) \widetilde{A}(\theta) y(\theta) d \theta+\int_{t}^{\infty} G(t, \theta) \widetilde{A}(\theta) y(\theta) d \theta\right) \\
& =A(t) X(t) \eta+\int_{t_{o}}^{\infty} A(t) G(t, \theta) \widetilde{A}(\theta) y(\theta) d \theta+G(t-0) \widetilde{A}(t) y(t)-G(t, t+0) \widetilde{A}(t) y(t) \\
& =(A(t)+\widetilde{A}(t)) y(t)
\end{aligned}
$$

and, for $t=t_{i}$,

$$
\begin{aligned}
y\left(t_{i}+0\right)= & X\left(t_{i}+0\right) \eta+\int_{t_{o}}^{\infty}\left(G\left(t_{i}+0, \theta\right) \widetilde{A}(\theta) y(\theta) d \theta\right. \\
& =B_{i} X\left(t_{i}\right) \eta+\int_{t_{o}}^{\infty} B_{i} G\left(t_{i}, \theta\right) \tilde{A}(\theta) y(\theta) d \theta=B_{i} y\left(t_{i}\right)
\end{aligned}
$$

Denote by $H$ the Banach space of all bounded piecewise continuous vector-valued functions $y(t)$ in the interval $\left[t_{o},+\infty\right)$ with points of discontinuity of the first kind at $t=t_{\boldsymbol{i}}, i=1,2, \ldots$ and with a norm $\|y\|=\sup _{t \geq t_{o}}|y(t)|$.

The linear operator

$$
\begin{gathered}
T y(t)=\int_{t_{o}}^{\infty} G(t, \theta) \widetilde{A}(\theta) y(\theta) d \theta \text { maps } H \text { into itself since } \\
|T y(t)| \leq \int_{t_{o}}^{\infty}|G(t, \theta)||\widetilde{A}(\theta)||y(\theta)| d \theta \leq N K\|y\| .
\end{gathered}
$$

This implies that $|T| \leq N K<1$ and by the contraction mapping principle the integral equation (2.6) for each $\eta \in Y$ has exactly one solution $y \in H$ which depends linearly on $\eta$, i.e. $y(t)=F(t) \eta$ where $F(t)$ is a bounded piecewise continuous matrix on the interval $\left(t_{o},+\infty\right)$ with points of discontinuity of the first kind at $t=t_{i}, i=1,2, \ldots$. Moreover, from $y=X(t) \eta+T y$, we obtain

$$
\|y\| \leq N|\eta|+|T|\|y\| \leq N|\eta|+N K\|y\|
$$

i.e., $\|y\| \leq \frac{N}{1-N K}|\eta|$ and $|F(t)| \leq \frac{N}{1-N K}$.

Let $\tilde{Y}$ be the subspace of $E$ consisting of the initial values of $y\left(t_{o}\right)$ of the bounded solutions of the integral equation (2.6)

where

$$
\begin{aligned}
y\left(t_{o}\right) & =\eta+\int_{t_{o}}^{\infty} G\left(t_{o}, \theta\right) \widetilde{A}(\theta) F(\theta) \eta d \theta \\
& =\eta-(I-P)\left(\int_{t_{o}}^{\infty} X^{-1}(\theta) \widetilde{A}(\theta) F(\theta) d \theta\right) \eta \\
& =(I-(I-P) Q P) \eta
\end{aligned}
$$

$$
Q=(I-P)\left(\int_{t_{o}}^{\infty} X^{-1}(\theta) \tilde{A}(\theta) F(\theta) d \theta\right) P .
$$


The operator $Q$ is bounded:

$$
|Q| \leq N K \frac{N}{1-N K}|P|
$$

The operator $I-(I-P) Q P$ maps the subspace $Y$ onto $\tilde{Y}$. It has a bounded inverse $I+(I-P) Q P$. The operator $\widetilde{P}=(I-(I-P) Q P) P(I-(I-P) Q P)^{-1}=P-(I-P) Q P$ is a projector with a range $R(\widetilde{P})=\widetilde{Y}$. The supplementary projector $I-\widetilde{P}=(I-P)(I+Q P)$ has a range $R(I-\widetilde{P})=Z$.

First we shall estimate the solutions issuing from $\tilde{Y}$. By (2.6)

$$
\begin{aligned}
\eta & =X^{-1}(s) y(s)-X^{-1}(s) \int_{t_{o}}^{\infty} G(s, \theta) \widetilde{A}(\theta) y(\theta) d \theta \\
& =X^{-1}(s) y(s)-\int_{t_{o}}^{s} P X^{-1}(\theta) \widetilde{A}(\theta) y(\theta) d \theta-\int_{s}^{\infty}(P-I) X^{-1}(\theta) \widetilde{A}(\theta) y(\theta) d \theta \\
& =P X^{-1}(s) y(s)-\int_{t_{o}}^{s} P X^{-1}(\theta) \widetilde{A}(\theta) y(\theta) d \theta
\end{aligned}
$$

i.e.,

$$
\begin{aligned}
y(t) & =X(t) \eta+\int_{t_{o}}^{\infty} G(t, \theta) \tilde{A}(\theta) y(\theta) d \theta \\
& =X(t) P X^{-1}(s) y(s)-X(t) \int_{t_{o}}^{s} P X^{-1}(\theta) \tilde{A}(\theta) y(\theta) d \theta+\int_{t_{o}}^{\infty} G(t, \theta) \tilde{A}(\theta) y(\theta) d \theta \\
& =X(t) P X^{-1}(s) y(s)+\int_{s}^{\infty} G(t, \theta) \tilde{A}(\theta) y(\theta) d \theta .
\end{aligned}
$$

Hence, for $t \geq s,|y(t)| \leq N|y(s)|+N \int_{s}^{\infty}|\tilde{A}(\theta)||y(\theta)| d \theta$

Let us fix $s$ and set $N|y(s)|=\alpha$. The cone of the nonnegative piecewise continuous functions

$\varphi(t)=|y(t)|$ is invariant with respect to the linear operator $L \varphi(t)=N \int_{s}^{\infty}|\tilde{A}(\theta)| \varphi(\theta) d \theta$.

Hence

$$
L|y(t)| \leq L \alpha+L^{2}|y(t)|=N K \alpha=N K L|y(t)|
$$

i.e.,

$$
|y(t)| \leq \alpha+L|y(t)| \leq \alpha+\frac{N K \alpha}{1-N K}=\frac{\alpha}{1-N K} .
$$

Hence for $t \geq s$

$$
|y(t)| \leq \frac{N|y(s)|}{1-N K}
$$

Let $z(t)$ be a solution with initial condition $z\left(t_{o}\right) \in Z$. From the formula

$$
z(s)=X(s) z\left(t_{o}\right)+\int_{t_{o}}^{s} X(s) X^{-1}(\theta) \tilde{A}(\theta) z(\theta) d \theta
$$

we express $z\left(t_{o}\right)$ and in view of $(I-P) z\left(t_{o}\right)=z\left(t_{o}\right)$ for $t<s$, we obtain that 


$$
\begin{aligned}
z(t) & =X(t)(I-P) X^{-1}(s) z(s)-\int_{t_{o}}^{s} X(t)(I-P) X^{-1}(\theta) \tilde{A}(\theta) z(\theta) d \theta \\
& +\int_{t_{o}}^{t} X(t) X^{-1}(\theta) \tilde{A}(\theta) z(\theta) d \theta \\
& =X(t)(I-P) X^{-1}(s) z(s)+\int_{t_{o}}^{t} X(t) P X^{-1}(\theta) \tilde{A}(\theta) z(\theta) d \theta \\
& -\int_{t}^{s} X(t)(I-P) X^{-1}(\theta) \tilde{A}(\theta) z(\theta) d \theta
\end{aligned}
$$

and get to the integral inequality

$$
|z(t)| \leq N|z(s)|+N \int_{t_{o}}^{s}|\tilde{A}(\theta)| z(\theta) \mid d \theta .
$$
The linear operator $L_{1} \varphi(t)=N \int_{t_{o}}^{s}|\tilde{A}(\theta)| \varphi(\theta) d \theta$ is monotone and, as for (2.7), we obtain that for
$t \leq s$

$$
|z(t)| \leq \frac{N}{1-N K}|z(s)|
$$

Now let $x(t)=y(t)+z(t)$ be an arbitrary solution of the differential equation (2.2). From the formula

$$
x(s)=X(s) x\left(t_{o}\right)+\int_{t_{o}}^{s} X(s) X^{-1}(\theta) \tilde{A}(\theta) x(\theta) d \theta
$$

we express $x\left(t_{o}\right)$ and in view of (2.6) we obtain that

$$
\begin{aligned}
y(s) & =X(s) P x\left(t_{o}\right)+\int_{t_{o}}^{\infty} G(s, \theta) \tilde{A}(\theta) y(\theta) d \theta \\
& =X(s) P X^{-1}(s) x(s)-\int_{t_{o}}^{s} X(s) P X^{-1}(\theta) \tilde{A}(\theta) x(\theta) d \theta \\
& +\int_{t_{o}}^{s} X(s) P X^{-1}(\theta) \tilde{A}(\theta) y(\theta) d \theta+\int_{s}^{\infty} X(s)(P-I) X^{-1}(\theta) \tilde{A}(\theta) y(\theta) d \theta \\
& =X(s) P X^{-1}(s) x(s)-\int_{t_{o}}^{s} X(s) P X^{-1}(\theta) \tilde{A}(\theta) z(\theta) d \theta \\
& +\int_{s}^{\infty} X(s)(P-I) X^{-1}(\theta) \tilde{A}(\theta) y(\theta) d \theta
\end{aligned}
$$

In view of (2.7) and (2.8) we get to the inequality

$$
\begin{aligned}
|y(s)| & \leq N|x(s)|+N \int_{t_{o}}^{s}|\widetilde{A}(\theta)||z(\theta)| d \theta+N \int_{s}^{\infty}|\tilde{A}(\theta)||y(\theta)| d \theta \\
& \leq N|x(s)|+\frac{N^{2} K}{1-N K}|z(s)|+\frac{N^{2} K}{1-N K}|y(s)| \\
& \leq \frac{N}{1-N K}|x(s)|+\frac{2 N^{2} K}{1-N K}|y(s)|,
\end{aligned}
$$

i.e.,

$$
|y(s)| \leq \frac{N}{1-N K-2 N^{2} K}|x(s)|
$$


Moreover,

$$
|z(s)| \leq|x(s)|+|y(s)| \leq \frac{1+N-N K-2 N^{2} K}{1-N K-2 N^{2} K}|x(s)| .
$$

From (2.7), (2.8), (2.9) and (2.10) we obtain that, for $t \geq s \geq t_{o},|y(t)| \leq N_{1}|x(s)|$, and, for $t \geq s \geq t_{o},|z(t)| \leq N_{1}|x(s)|$ where $N_{1}=\frac{N\left(1+N-N K-2 N^{2} K\right)}{(1-N K)\left(1-N K-2 N^{2} K\right)}>0$, i.e., the perturbed equation (2.2) has an ordinary dichotomy as well.

LEMMA $1([8])$. Let $\tau_{o}$ and $\tau_{o}$ be fixed real numbers in the interval $\left[t_{o},+\infty\right)$ and let the impulse matrices $B_{i}, i=1,2, \ldots$, of equation (2.1) be nonsingular. If equation (2.1) has an ordinary dichotomy on the interval $\left[\tau_{o},+\infty\right)$, then it has an ordinary dichotomy on the interval $\left[\tau_{o},+\infty\right)$ as well.

COROLLARY 1. Let the impulse matrices $B_{i}, i=1,2, \ldots$, be nonsingular and let equation (2.1) have an ordinary dichotomy on the interval $\left[t_{o},+\infty\right)$. If

$$
\int_{t_{o}}^{\infty}|\tilde{A}(\theta)| d \theta<\infty
$$

then the perturbed equation (2.2) also has an ordinary dichotomy on the interval $\left[t_{o},+\infty\right)$.

PROOF. Since the integral $\int_{t_{o}}^{\infty}|\widetilde{A}(\theta)| d \theta$ is convergent, then a sufficiently large number $\tau_{o}$ can be found such that condition (2.5) should hold. Since the impulse matrices $B_{i}, i=1,2, \ldots$, are nonsingular, then equation (2.1) has an ordinary dichotomy with the same constant $N$ on the interval $\left[\tau_{o},+\infty\right)$ as well. Then by Theorem 1 the perturbed equation (2.2) also has an ordinary dichotomy on the interval $\left[\tau_{o},+\infty\right)$ and by Lemma 1 it has an ordinary dichotomy on each interval $[\tau,+\infty), \tau \geq t_{o}$ as well.

ACKNOWLEDGEMENT. The present investigation is supported by the Ministry of Culture, Science and Education of People's Republic of Bulgaria under Grant 61.

\section{REFERENCES}

1. COPPEL, W.A. Dichotomies in Stability Theory, Lecture Notes in Math., Springer-Verlag, 629 (1978).

2. DALECKII, JU. L. and KREIN, M.G. Stability of Solutions of Differential Equations in Banach Spaces, Trans.Amer.Math.Soc., Providence, R.I. (1974).

3. DISHLIEV, A.B. and BAINOV, D.D. Continuous dependence of the Solution of a System of Differential Equations with Impulses on the Impulse Hypersurfaces, J.Math.Anal.Appl. Vol. $\underline{135}$ №. 2 (1988), 369-382.

4. HEKIMOVA, M.A. and BAINOV, D.D. Periodic Solutions of Singularly Perturbed Systems of Differential Equations with Impulse Effect, ZAMP, Vol. 36 (1985), 520-537.

5. LAKSHMIKANTHAM, V. and LIU, XINZHI Stability for Impulsive Differential Systems in Terms of Two Measures, Appl.Math.Comp. (to appear).

6. LAKSHMIKANTHAM, V. and LIU, XINZHI On Quasi Stability for Impulsive Differential Systems, Nonlinear Analysis (to appear).

7. MASSERA, J.L. and SCHÄFFER, J.J. Linear Differential Equations and Functional Analysis, $\underline{\mathrm{I}}$ Ann. of Math. $\underline{67}$ (1958), 517-573.

8. MILEV, N.V. and BAINOV, D.D. Dichotomies for Linear Differential Equations with Variable Structure and Impulse Effect (to appear).

9. PALMER, K.J. A Perturbation Theorem for Exponential Dichotomies, Proc.Roy.Soc. Edinburgh $106 \mathrm{~A}$ (1987), 25-37.

10. SIMEONOV, P.S. and BAINOV, D.D. Stability with Respect to Part of the Variables in Systems with Impulse Effect, J.Math.Anal.Appl., Vol. 117, No. 1 (1986), 247-263. 


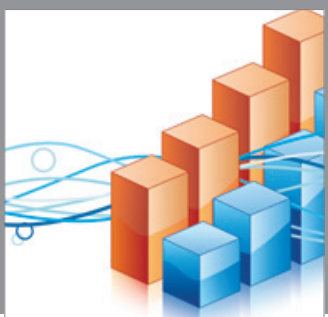

Advances in

Operations Research

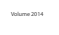

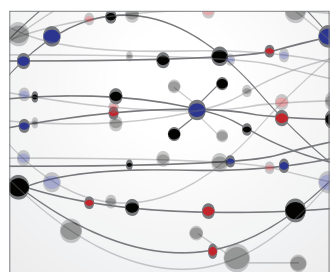

\section{The Scientific} World Journal
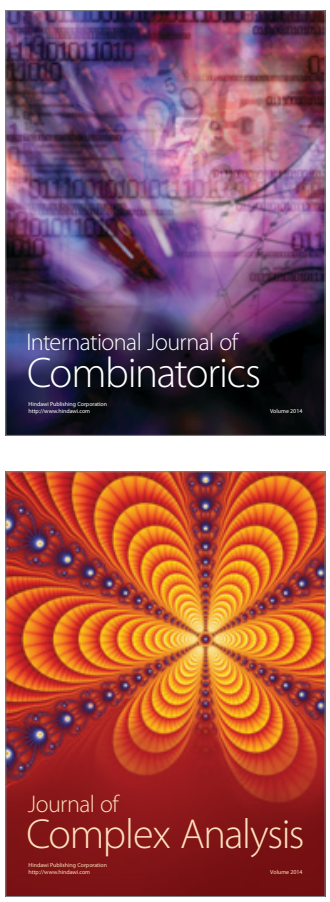

International Journal of

Mathematics and

Mathematical

Sciences
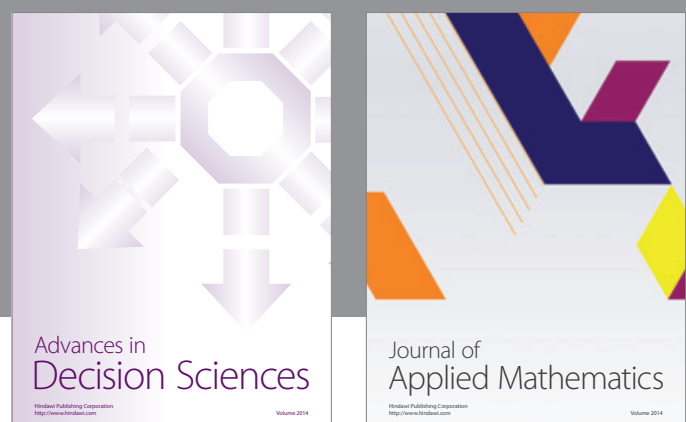

Journal of

Applied Mathematics
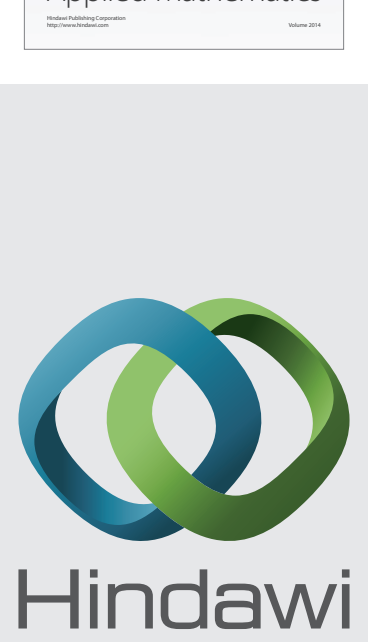

Submit your manuscripts at http://www.hindawi.com
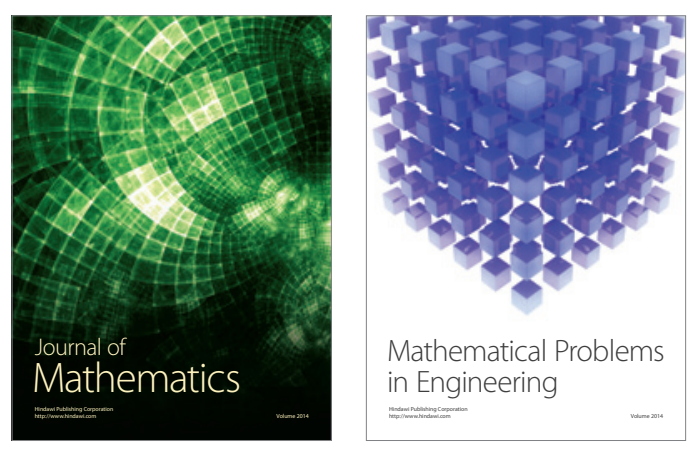

Mathematical Problems in Engineering
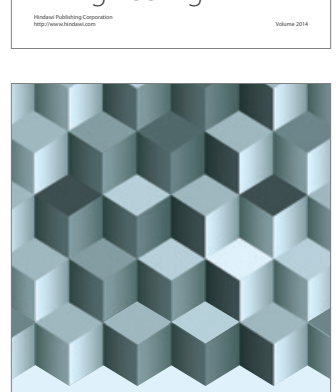

Journal of

Function Spaces
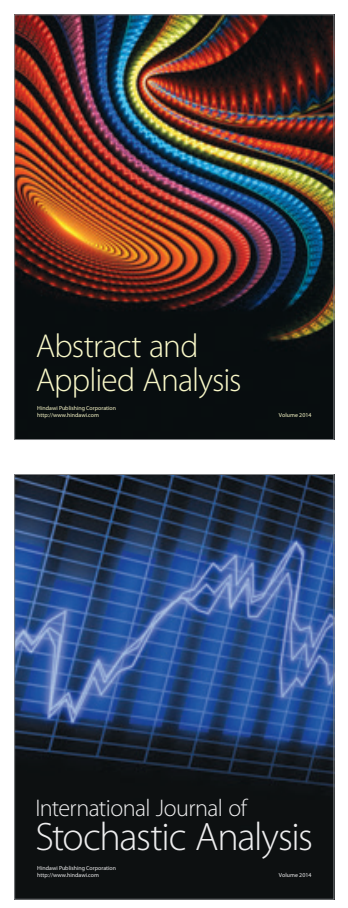

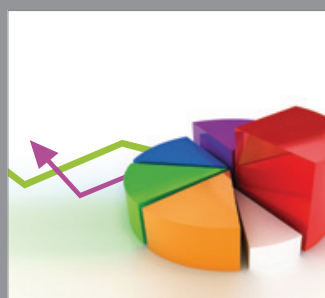

ournal of

Probability and Statistics

Promensencen
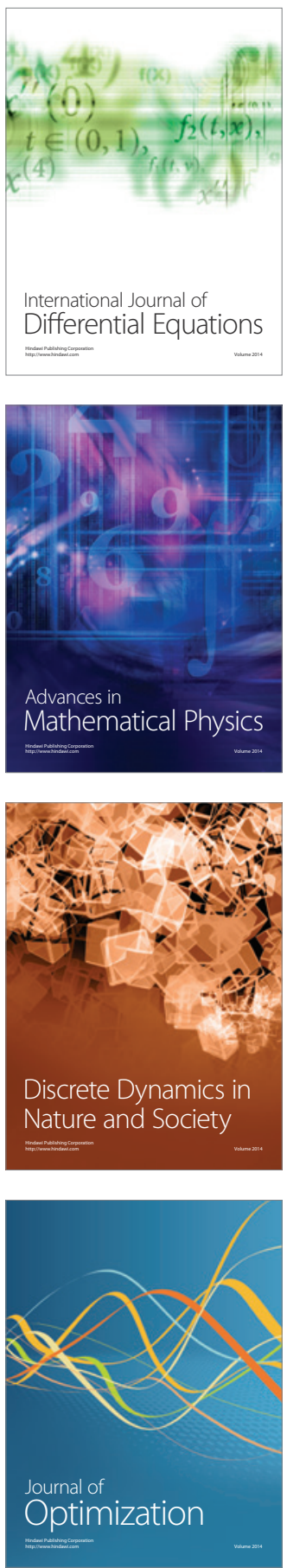